\title{
Experimental Study on Net Flow Rate Generation Inside Closed Loop Mechanical Circulatory System Using Impedance Pump
}

\author{
Azli Abd Razak ${ }^{1 *}$, Nazirul Izzati Rosli ${ }^{1}$, Mohd Faizal Mohamad ${ }^{1}$

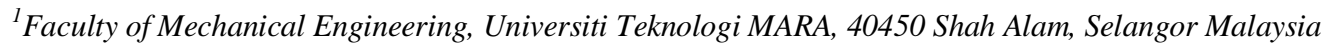 \\ *Corresponding author E-mail:azlirazak@salam.uitm.edu.my
}

\begin{abstract}
This present study focused on the performance of net flow rate inside closed loop mechanical circulatory system with single and double pinching impedance pumps which generates a unidirectional flow of fluid around closed loop of soft viscoelastic tubing. The experimental setup consisted of viscoelastic tubing connected between two ends of rigid tube which was compressed rhythmically or squeezed asymmetrically at various frequencies by motorized pinching. Hence, net flow of fluid around the tubing can occur without valves. Experiment was done on two different fluid, namely Newtonian and Non-Newtonian. Result showed that the flow rate inside closed loop system for non-Newtonian fluid and Newtonian fluid were in good agreement with each other. Single pinching showed a lower flow rate compared to double pinching at higher frequency. The results could be used as a model for a new Mechanical Circulatory Support System used by cardiac patients. Factors influencing the performance of valveless impedance pump was also explained.
\end{abstract}

Keywords: Impedance Pump; Close Loop; MCS; Flow Rate; Non-Newtonian

\section{Introduction}

Heart disease or coronary heart disease is the world top killer contributing to a higher mortality rate each year with high cost of treatment and medication [1]. In 2014, Ministry of Health Malaysia recorded that $6.99 \%$ of total hospital admissions and $23.34 \%$ of all hospital deaths in 2014 were due to circulatory disease [2]. According to the World Health Organization, circulatory disease was the most common cause of death in Malaysia in 2012.

There are several treatments for heart failure depending on the stages of the disease. The most efficient treatment to raise the survivability of the advance heart failure is the Ventricular assist device (VAD) [3]. This implantable durable VAD is a Mechanical circulatory system (MCS) is an alternative to cardiac transplantation for patients with advance heart failure [4]. VAD is one of the most efficient treatments to raise the survivability of patients at final stage of their heart failure.

The function of VAD is to support dysfunctional heart during recovery process, as well as to allow the heart to be relieved from workload. MCS is a continuous-flow pump to keep the blood flow continuously. Some patients may be able to do life routine as usual while letting the heart to heal. Eventually, the patients will be able to live without MCS and escape a heart transplant. MCS can also maintain the oxygenated blood circulations that pump the blood from the heart through the body. The human heart is the most efficient valves where it will ensure smooth blood flows from the heart into arteries and that of returned by veins.

Motivated by the need for MCS in advance heart failure treatment, the present study aims to analyze the flow rate inside the closed loop system at different location and tube diameter. Specifically, the effect of mechanical system on the flow rate inside the MSC is classified into two: pinching activities and location, and internal diameter of elastic tube. In the present work, we use a valveless pump similar to that used by Liebau [5]. The mechanical forcing mechanism is used to pinch the elastic tube at various frequencies and locations. Subsequently, the effect of elastic tube diameter is determined through three different sizes of elastic tube inner diameter.

The paper is divided into four main sections. Section 1 covers the introduction and history of impedance pump. Section 2 describes the details of experimental setup and procedure. The effect of various parameters on mean flow rate is examined extensively in Section 3. Finally, Section 4 discusses and concludes results on the correlation between flow rate and mechanical parameters (pinching stages, location, and elastic tube diameter).

\subsection{Impedance Pump}

Impedance pump is defined as a type of valveless pumping mechanism, where an elastic tube is joined with a rigid tube of different impedance. An asymmetrical compression is continuously applied on the elastic tube part, which is able to produce wave reflection and unidirectional flow. Elastic tube is made up of materials such as rubber, silicone, latex and other elastic materials which can transfer energy greatly to propagate the wave. Liebau [5] was the first person who demonstrated the experiment on the elastic tube with reservoir which also known as open loop system. He revealed that flow of fluid can occurred by periodic compression of the tube. In addition, he found that the movement of the fluid always travel from the longer section toward shorter section when compression was applied to the asymmetric tube. Rhythmically pinching stages would generate unidirectional flow across the model. The flow relied fully on the pinching location whether it was clockwise or anti clock wise for the closed loop model [6].

Generally, model of impedance pump consists of elastic tube and a rigid tube filled with fluid as shown in Fig. 1. Fluid will flow uni-directionally by external pinching and thus, there is no use of moving blade. A unidirectional flow created by rhythmically pinching is called Liebau phenomenon or valveless pumping $[1,5]$ Many researchers focused into simulation studies where different cases of pump structures, tube parameters can be tested repeatedly. 
However, the result obtained needs to be validated with the experimental data. Impedance pump is still on subjective deliberation and starting to gain interest due to its potential. Due to its intricate nature and reliance on some parameters, many researchers have simplified its model from various numerical studies [7-10] and identified the parameters and documented the behavior of the flow from experimental studies [11-13].

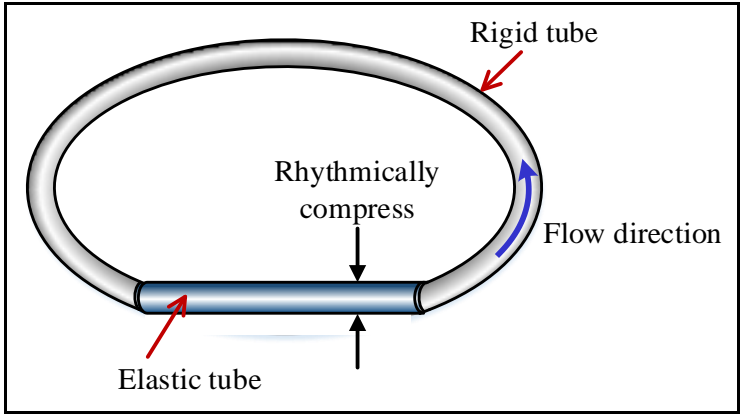

Fig. 1: Closed loop impedance pump

\section{Experiment Setup}

Fig. 2 shows an experimental setup that consists of two differen tubes filled with fluid in a close loop system. A DC motor was used for the mechanical output to continuously pinch the elastic tube at desired speed. The voltage regulator functioned as power supply as well as voltage and current controller for the DC motor. For stable rotation of current, it was adjusted to the maximum on the regulator and the voltage was varied. During the experiment, current value kept changing as it carried the load where in this experiment the load was equal to the pincher. Elastic tube was pinched rhythmically at one point resulting fluid flow around the loop. Flow meter was attached as in Fig. 2 to measure the flow.

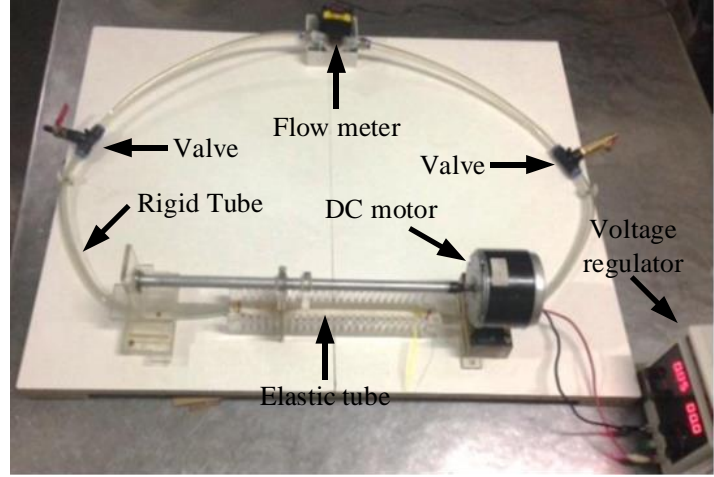

Fig. 2: Experiment set up

Tubing consists of elastic and rigid tubes with total length of 194 $\mathrm{cm}$. Polyurethane tube was used for the rigid tube while latex balloon was used for the elastic tube. Four different diameters of elastic tube were tested. Detailed parameters on rigid and elastic tubes were listed in the Table 1 .

Pincher holder was designed with slots to guide and hold the moving pincher during experiment as illustrated in Fig. 3 to ensure that the pinching position was repeated at the same position. For double stages pinching, two pinchers were attached side by side in the slots where each slot was $5 \mathrm{~mm}$ apart. The length of the pincher holder was equal to the length of elastic tube which was $220 \mathrm{~mm}$. The elastic tube diameter was $32 \mathrm{~mm}$ at rest.

For the pinching location along an elastic tube, it was divided into two parts which were labelled as L0 and L25 as shown in Fig. 4. Pinching on the center of the tube resulted in zero flow hence to avoid zero condition, both positions were selected to perform the observation. The measurement of L0 position was $110 \mathrm{~mm}$ away from center, while L 25 was $55 \mathrm{~mm}$ from center.

Fig. 5 is a schematic diagram of double pinching position. The angle of each cam is $90^{\circ}$. As the power is switched on, shaft would rotate together at y axis. This cam would rotate and simultaneously push downward the pinchers. The rotational speed depends on voltage value. Higher voltage would lead to increased rotation per second (Frequency, Hz). Each rotation would drive the pincher to move downward; as a result it would pinch the elastic tube and create flow through the loop.

Table 1: Rigid and elastic tube specification

\begin{tabular}{|l|c|c|}
\hline \multicolumn{1}{|c|}{ Tube description } & Elastic \\
\hline Inner diameter & Min 6 mm, Max 32 mm & \multicolumn{1}{c|}{ Rigid } \\
\hline Thickness & 0.2 to $0.3 \mathrm{~mm}$ \\
\hline Length & $220 \mathrm{~mm}$ & $10 \mathrm{~mm}$ \\
\hline Elasticity, young modulus & 2 to $3 \mathrm{MPa}$ & $1720 \mathrm{~mm}$ \\
\hline
\end{tabular}

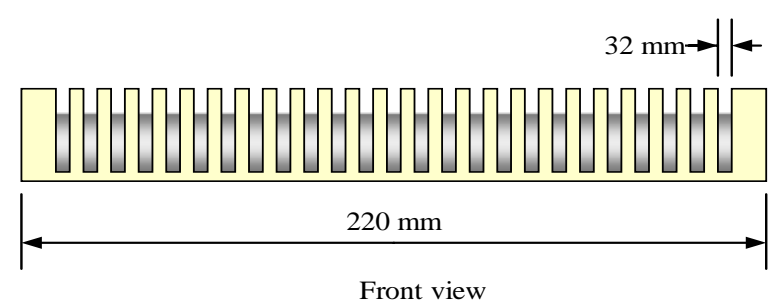

Fig. 3: Pincher holder

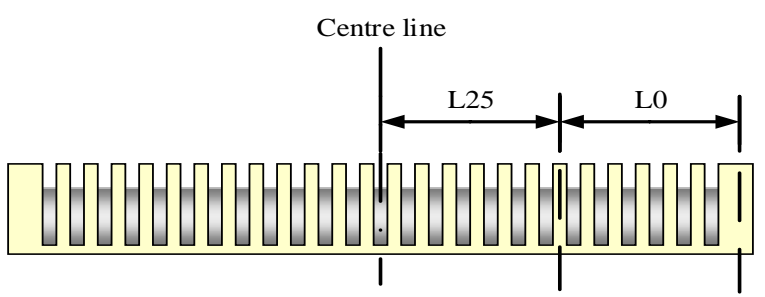

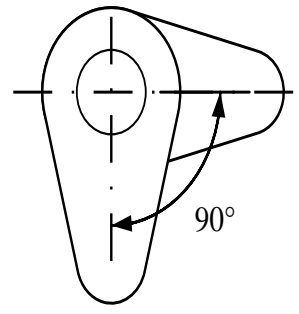

Side view

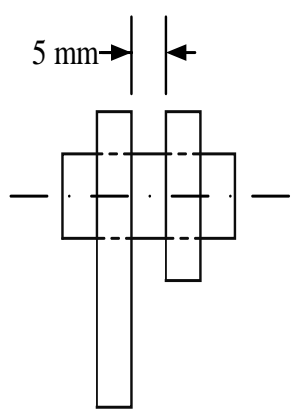

Front view

Fig. 5: Schematic drawing of double pincher 


\subsection{Experimental Procedure}

The test was performed at room temperature on a flat, smooth and leveled surface. The close loop impedance pump arrangement was a soft tube section connected to a rigid tube and a flow meter. Fluid was inserted using a hand pump at one of the valve, while the other remained closed. A pump was used to create sufficient flow over air resistance in the close loop tubing. For the fluid inlet and outlet, two $6.5 \mathrm{~mm}$ ball valves were attached on both sides to remove the trapped air inside the tube. Bubbles- and leakage-free were important to maintain steady flow, thus avoiding error for flow meter. When the loop was completely filled with fluid, it was ready to be pinched.

The pinching stages on the elastic tube generated one way pulsating flow around $194 \mathrm{~cm}$ circumference of closed loop system. The pincher width was $2 \mathrm{~cm}$ and pinching stages occurred at two positions: $11 \mathrm{~cm}(\mathrm{~L} 0)$ and $55 \mathrm{~cm}(\mathrm{~L} 25)$ away from the middle of elastic tube.

\section{Result}

The graph of flow rate against frequency is presented for three different fluids for dual pinching. A $35 \mathrm{~mm}$ diameter elastic tube is utilized to observe the flow, preliminary. All values taken were in positive region as the flow travelled clockwise. Pinching at the middle section of elastic tube results in zero flow. Fig. 6 shows that flowrates increases as frequency increases. The trend is observed for all fluid solutions. Water and Glycerine solution mixture were used and the Glycerine content was measured by percentage. The initial flowrate recorded is $40 \mathrm{ml} / \mathrm{min}$ which increases to about $140 \mathrm{ml} / \mathrm{min}$ at frequency of $1.8 \mathrm{~Hz}$ and $7.3 \mathrm{Gz}$, respectively.

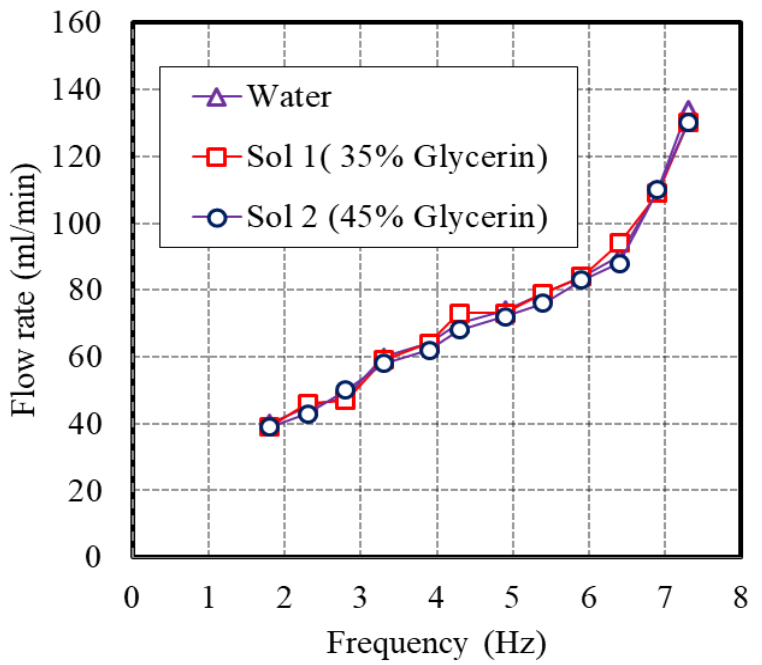

Fig. 6: Water and Glycerine tested with $35 \mathrm{~mm}$ diameter of elastic tube at L0 position, $110 \mathrm{~mm}$ away from the centre with double pinching.

Fig. 7 (a) and (b) show the flow rate against frequency for different pinching conditions using $35 \mathrm{~mm}$ diameter tube. Both results show an increment of flow rate with frequency. The flowrate for single compression for both positions increases steadily but for double compression the flow starts to increase rapidly after $3.9 \mathrm{~Hz}$ at position $\mathrm{L} 0$ and $2.8 \mathrm{~Hz}$ at position $\mathrm{L} 25$ due to the increase of the pinching cycle. In addition, the flowrate for single compression increases gradually at position L25. The flowrate for single compression starts at $33 \mathrm{ml} / \mathrm{min}$ and increases to $76 \mathrm{ml} / \mathrm{min}$ (Fig. 7(a)) and starts at $34 \mathrm{ml} / \mathrm{min}$ and increases up to $57 \mathrm{ml} / \mathrm{min}$ (Fig. 7(b)). This implied that the pinched position near to the centre may cause reduction in the flow rate.

Fig. 8 (a), (b) and (c) show the comparison of different pipe diameters measured at L25. It is obvious that the flowrate is lower for smaller diameter pipe in comparison to that of a bigger diameter pipe. In addition, for single pinch condition of the $10 \mathrm{~mm}$ diameter pipe, the fluid only starts to flow at frequency $>2 \mathrm{~Hz}$ whereas that of other diameters flows at $<2 \mathrm{~Hz}$. Also, the single pinching flows at higher frequency compared to the double pinching. This result implies that a small diameter tube needs higher frequency to flow the fluid inside the pipe using impedance pump. Fig. 8(d) shows the $\mathrm{R}^{2}$ for all diameters of pipes. All $\mathrm{R}^{2}$ shows a positive value near 1 . This value indicates that the diameter of pipe has a strong correlation with flow rate and pinching frequency.

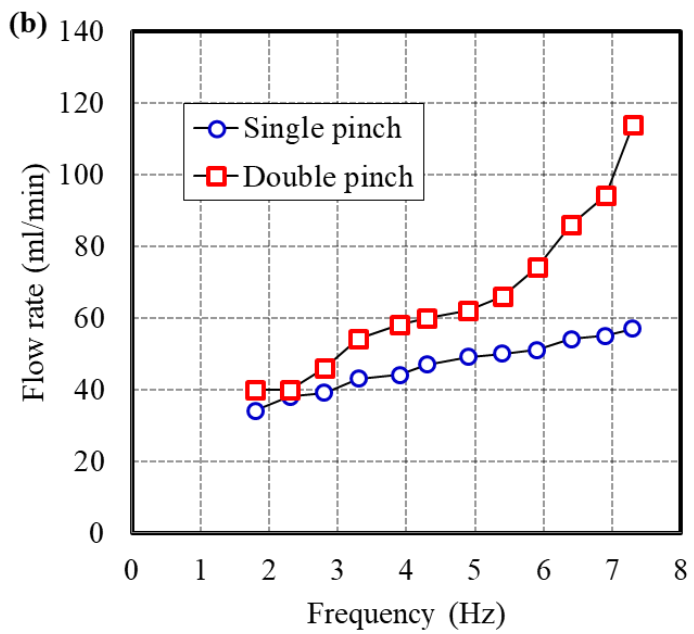

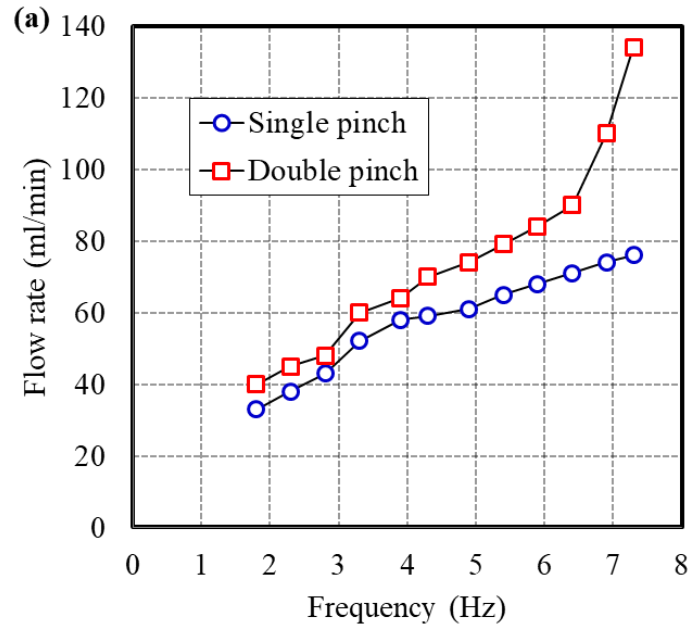

Fig. 7: Mean flow rate versus frequency with $35 \mathrm{~mm}$ diameter of elastic pipe.(a) pinching at location L0 and (b) pinching at location L25. 

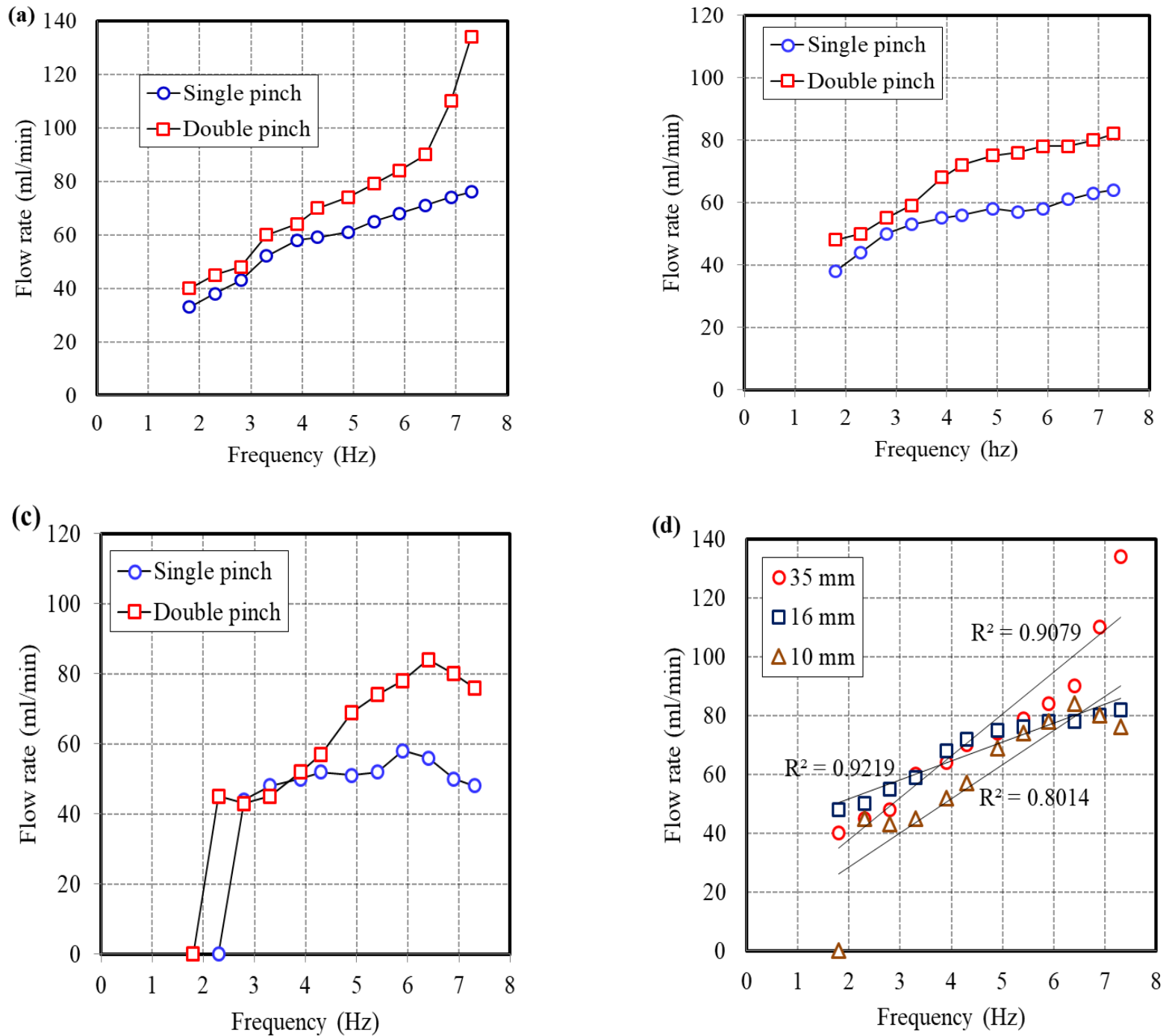

Fig. 8: Mean flow rate versus frequency at pinching location L25. (a) $35 \mathrm{~mm}$ pipe diameter, (b) $16 \mathrm{~mm}$ pipe diameter, (c) $10 \mathrm{~mm}$ pipe diameter and (d) R-square

\section{Conclusion}

A fluid filled closed loop system was tested with different fluid on two distinct positions; L0 and L25. First experiment was tested in single pinching condition and then it was repeated in double pinching. For all the fluids tested; water, water $+35 \%$ Glycerine and water $+45 \%$ Glycerine, there was not much difference in the flow rate. The flowrate for all the fluids was almost the same, thus, it is more convenient to select water as a medium to run an experiment on impedance pump system. Single and double pinching showed a strong effect on the flow rate, however, the flowrate for single pinching was less than that of the double pinching. This may be due to the double pinching has twice compression time in one cycle. From this experiment, it also demonstrates that by using single motor, the flow in the system still can be increased at the same voltage of single pinching instead of using double motor. In addition, the diameter of elastic pipe shows a strong correlation o flow rate and frequency. It is clear that design parameters such as tube diameter and pinching conditions are significant in designing the closed loop impedance pump, which are sometimes overlook.

\section{Acknowledgement}

This work was supported by Ministry of Higher Education under Fundamental Research Grant Scheme through the Research Man- agement Institute, Universiti Teknologi MARA (600-IRMI / GIP $5 / 3(0045 / 2016)$. Authors also acknowledges the used of facilities in Faculty of Mechanical Engineering, Universiti Teknologi MARA, Shah Alam, Malaysia.

\section{References}

[1] Mozaffarian, D., et al., AHA Statistical Update Heart Disease and Stroke Statistics-2016 Update A Report From the American Heart Association WRITING GROUP MEMBERS. 2016.

[2] Seong, A.C. and C.K.M. John, A Review of Coronary Artery Disease Research in Malaysia. Med J Malaysia, 2016. 71: p. 43.

[3] Kim, Y.I., et al., Estimation of ventricular assist device outflow with the pressures in air pressure line. Journal of Biomedical Engineering Research, 2014. 35(5): p. 119-124.

[4] Slaughter, M.S. and R. Singh, The Role of Ventricular Assist Devices in Advanced Heart Failure. Revista Española de Cardiología, 2012/11/01/ 2012. 65(11): p. 982-985.

[5] Liebau, G., Über ein ventilloses pumpprinzip. Naturwissenschaften, 1954. 41(14): p. 327-327.

[6] Kenner, T., et al., The Liebau-effect or on the optimal use of energy for the circulation of blood. Scripta Medica (BRNO), 2000. 73: p. 9-14.

[7] Avrahami, I. and M. Gharib, Computational studies of resonance wave pumping in compliant tubes. Journal of Fluid Mechanics, 2008. 608: p. 139-160.

[8] Ibrahim, M.S.B. and M.M.B. Mahat, CFD analysis of electromagnetic based valveless pump. Procedia Engineering, 2012. 41: p. 1524-1532. 
[9] Shin, S.J., C.B. Chang, and H.J. Sung, Simulation of a valveless pump with an elastic tube. International Journal of Heat and Fluid Flow, 2012. 38: p. 13-23.

[10] Shin, S.J. and H.J. Sung, Three-dimensional simulation of a valveless pump. International Journal of Heat and Fluid Flow, 2010 31(5): p. 942-951.

[11] Babbs, C.F., Behavior of a viscoelastic valveless pump: a simple theory with experimental validation. Biomedical engineering online, 2010. 9(1): p. 42.

[12] Mahat, M.M., et al. Impedance Induce Valve Pump in Closed Loop System. in Applied Mechanics and Materials. 2013. Trans Tech Publ.

[13] Ibrahim, M.H. and M. Al-Atabi, Effect of Viscosity on Impedance Pump Performance, in Engineering Undergraduate Research Catalyst Conference. 2013: Taylor University, Malaysia. p. 91-92. 